\title{
Tagungsbericht
}

Vivien Petras* und Stefan Schmunk

\section{Digitale Lehre in der Informationswissenschaft - Von der Pandemie bedingte Notwendigkeit zu Lessons Learned und Best-Practices}

\author{
Bericht zu den Digitalen Workshops am 25. September und 2. Oktober 2020
}

https://doi.org/10.1515/iwp-2020-2128

Die Corona-Pandemie und die damit einhergehenden Einschränkungen bei der Durchführung von Präsenz-Veranstaltungen haben bundesweit dazu geführt, dass im März 2020 oftmals ad-hoc und rasch bereits geplante Veranstaltungen für das Sommersemester auf digitale Formate umgestellt werden mussten. Dieses „Corona-Semester” stellte sicherlich nicht nur für die informationswissenschaftlichen Studiengänge eine Herausforderung dar, sondern war zugleich eine Chance, in der oftmals kreativ und innovativ digitales Lehren und Lernen realisiert wurde. Aufgrund der pandemischen Entwicklung von Covid-19 werden auch das anstehende Wintersemester und wahrscheinlich auch das Sommersemester 2021 an den meisten Hochschulen ebenfalls digital oder in hybriden Veranstaltungen durchgeführt. Die Workshops hatten zum Ziel, Best-Practices und Lessons-Learned aus der Community mit der Community auszutauschen.

Der „Hochschulverband Informationswissenschaft (HI)” und die „Konferenz der informations- und bibliothekswissenschaftlichen Ausbildungs- und Studiengänge (KIBA)", Sektion 7 des dbv und Ausbildungskommission der DGI, griffen deshalb gemeinsam diese Thematik auf und befassten sich im Rahmen zweier virtueller Workshops, welche Schlüsse für die Informationswissenschaft daraus zu ziehen sind. Hierbei wurde diskutiert, inwiefern digitale Methoden, Konzepte und didaktische Ansätze auf Dauer in einer informationswissenschaftlichen Lehrkultur aufgenommen werden können. Während beim ersten Workshop vor allem der Themenkomplex „Lessons Lear-

\footnotetext{
*Kontaktperson: Prof. Vivien Petras, PhD, Humboldt-Universität zu Berlin, Institut für Bibliotheks- und Informationswissenschaft, Lehrund Forschungsbereich Information Retrieval, Dorotheenstraße 26, 10117 Berlin, E-Mail: vivien.petras@ibi.hu-berlin.de

Prof. Dr. Stefan Schmunk, Hochschule Darmstadt, Fachbereich Media, Max-Planck-Straße 2, 64807 Dieburg, E-Mail: stefan.schmunk@h-da.de
}

ned" im Mittelpunkt stand, wurden beim zweiten Workshop Anfang Oktober Beispiele für didaktischen Konzepte und Fallbeispiele vorgestellt und diskutiert.

Die Vorträge sind als Video und Folienpräsentation persistent auf dem Medienrepositorium der HumboldtUniversität zu Berlin abrufbar. ${ }^{1}$

\section{Workshop I: Lessons Learned im Sommersemester 2020}

1. Dr. Ulrike Wuttke / Prof. Dr. Heike Neuroth (FH Potsdam): Weiterbildender Master „Digitales Datenmanagement - DDM“: Corona Lockdown, digitaler Start \& Lessons Learned

2. Prof. Dr. Monika Steinberg / Dr. Anke Wittich / Gerrit Wucherpfennig (Hochschule Hannover): Do you need it perfect or by Tuesday? Videos in der digitalen Lehre

3. Dr. Ulla Wimmer (HU Berlin): Von wegen ,any time, any place“ - Zeit, Raum und Struktur in der digitalen Lehre

4. Julian Hocker / Carolin Keller / Verena Weimer / Prof. Dr. Marc Rittberger (DIPF / Hochschule Darmstadt): Transformation ins Digitale

Der erste Workshop zur digitalen Lehre in der Informationswissenschaft am 25. September 2020 wurde von 51 Teilnehmenden in der Zoom-Konferenz besucht. Jedem Vortrag folgte eine spannende Diskussion, die mit zusätzlichen Wortmeldungen im Chat noch stärker bereichert wurde. Ulrike Wuttkes Vortrag zur Einführung und Betreuung im weiterbildenden Masterstudiengang „Digitales

$1 \mathrm{http} / / /$ www.informationswissenschaft.org/allgemein/videos-undpraesentationen-der-workshops-digitale-lehre-in-der-informations wissenschaft-nun-verfuegbar/[30.11.2020]. 
Datenmanagement- DDM“ förderte insbesondere die Diskussion, wie der soziale Zusammenhalt und die Kohärenz in einer Studierendengruppe bzw. Kohorte in Lehrveranstaltungen bzw. Studiengängen in einer Online-Lernumgebung entwickelt und verstärkt werden könnte. Vorgestellt wurde u.a. das Stimmungsbarometer, entwickelt unter der Leitung von Kai Eckert an der HdM Stuttgart. ${ }^{2}$ Ein weiteres Werkzeug ist die wonder Plattform ${ }^{3}$ (ehemals Yotribe), die es erlaubt, virtuelle Räume zu gestalten, in denen man sich zwangslos begegnen kann. Komplementäre Treffen im analogen Bereich wurden aber als sehr wichtig eingeschätzt.

In Ihrem Vortrag betonten Anke Wittich und Gerrit Wucherpfennig von der Hochschule Hannover, dass qualitativ hochwertige Lehrressourcen (wie z. B. das interaktive Lehrvideo zur Funktion von Suchmaschinen ${ }^{4}$ ) entsprechende Ressourcen benötigen und dass „low fidelity“ Aufnahmen von Lehrveranstaltungen auch im Portfolio der Ressourcen enthalten sein können. Kritisiert wurde der sog. „Tool-Zoo“, dem sich Studierende in der Online-Umgebung gegenüberstehen, wobei eine optimale Lösung noch aussteht. Die Diskussion fokussierte darauf, welche Arten von Lehrressourcen effektiv sind (asynchrone Videos, Aufnahmen von synchronen Lehrveranstaltungen, Foliencasts, Quizze) und inwiefern die Veröffentlichung als OER (Open Educational Resources) ein Weg für die informationswissenschaftlichen Hochschulen sein könnte, höherwertiges Lehrmaterial anzubieten und sich gegenseitig besser auszutauschen.

Ulla Wimmers Vortrag von der Humboldt-Universität $\mathrm{zu}$ Berlin diskutierte die zeitlichen Herausforderungen, die sich aus dem „Flipped Classroom“-Modell ergeben. Dieser Ansatz war am Institut für Bibliotheks- und Informationswissenschaft für alle Kurse vorgegeben (Prinzipien: alle relevanten Inhalte werden asynchron zur Verfügung gestellt, Online-Sitzungen sollen für Fragen und Diskussionen verwendet werden). Studierende und Lehrende empfinden das Modell gleichermaßen als aufwendiger, da es zwar an einigen Stellen mehr Flexibilität erlaubt, oft aber auch mehr Zeit bindet. Die Diskussion erörterte Vorschläge, wie man die Struktur klar kommunizieren könnte, aber auch, wie im kommenden Wintersemester Präsenz- und Onlinelehre miteinander kombiniert werden könnten.

In ihrem Vortrag über zwei Online-Kurse an der Hochschule Darmstadt gingen Julian Hocker und Carolin Keller

2 http://barometer.wisslab.org/ [25.10.2020].

3 https://www.wonder.me/ [25.10.2020].

4 https://q-plus-im.wp.hs-hannover.de/einblick-in-die-suchmaschi nen-welt-aus-duplo-steinen/ [25.10.2020]. auf die verschiedenen Lehr- und Lernelemente ein, die sie für die Vermittlung der Inhalte und insbesondere für die Kommunikation und den Austausch mit den Studierenden einsetzten. In der Diskussion wurde viel über die Frage diskutiert, wie man Studierende aktivieren könnte und welche Interaktionen gut im Online-Raum funktionierten, um den virtuellen Austausch zu verstärken. Kleinere Gruppen, persönliche Ansprechformen und Aktivierungen, die im Chat funktionieren, wurden genannt.

\section{Workshop II: Best Practices mit Fallbeispielen}

1. Prof. Dr. Jutta Bertram (Hochschule Hannover): Screencasts und Audio-Instruktionen, Übungen und kommentierte Musterlösungen - Ein asynchrones Online-Lehrkonzept für die Inhaltserschließung

2. Prof. Dr. Thomas Mandl / Dr. Stefan Dreisiebner (Universität Hildesheim): Von digitaler Lehre im Sommersemester zu nachhaltigen Open Educational Resources: Das Beispiel des Seminars Informationsethik an der Universität Hildesheim

3. Hannes Schnaitter, M. A. (HU Berlin): Ein Gegenüber bieten - Moderation zur Unterstützung der OnlineLehre

4. Nico Saß, M. A. (HU Berlin): „Ich habe permanent das Gefühl, dass ich nicht Schritt halten kann.“ - Feedback zur Lehre in einem komplett digitalen Semester

Der zweite Workshop fand am 2. Oktober 2020 statt und widmete sich dem Themenkomplex „Best Practices und Fallbeispiele“. Die vier Vorträge führten unter den 54 Teilnehmenden zu lebhaften Diskussionen und identifizierten über die einzelnen Vorträge hinaus weitere Themen, die für die Durchführung von virtuellen synchronen sowie asynchronen Lehrveranstaltungen von Bedeutung sind. Jutta Bertram von der Hochschule Hannover stellte in ihrem Vortrag ein Online-Lehrkonzept vor, bei dem mittels Audio-Instruktionen und 10- bis 15-minütigen Screencasts das Thema Inhaltserschließung vermittelt wurde. Bei der Lehrveranstaltung mit ca. 40 Studierenden im zweiten Semester lag der Schwerpunkt auf einer asynchronen Vermittlung, wobei zugleich der wöchentliche Rhythmus einer virtuellen Präsenz-Veranstaltung beibehalten wurde. Mittels Quiz und dazugehöriger kommentierter Lösungen erhielten die Studierenden die Möglichkeit die erlernten Fähigkeiten während des Semesters selbständig zu prüfen und erhielten über Moodle individuelles Feedback. An den Vortrag schloss sich eine Diskussion über die Proble- 
me der Übertragbarkeit von Teamaufgaben und -arbeiten in den virtuellen Raum an und es wurde debattiert, wie bei asynchroner Lehre die Motivation von Studierenden aufrechterhalten bzw. ausgebaut werden kann.

Stefan Dreisiebner von der Universität Hildesheim gab anhand des Fallbeispiels der englischsprachigen Lehrveranstaltung „Information and Society: Information Ethics“ einen Einblick in die Transformation dieser bislang in Präsenz durchgeführten Veranstaltung zu einem Online-Seminar, das über Moodle durchgeführt und direkt nach Abschluss dauerhaft als OER-Ressource zur Verfügung gestellt wurde. Auch in dieser Lehrveranstaltung wurde nach jedem Themenblock ein Online-Quiz durchgeführt und zugleich wöchentliche Online-Präsenz-Sitzungen abgehalten, um organisatorische und inhaltliche Diskussionen zu ermöglichen. Die Studierenden mussten eigenständig Inhalte als Screencasts erarbeiten, die von ihren Kommilitonen begutachtet wurden. Diese Rückmeldung wurden mit dem Feedback der Lehrenden kombiniert. Das Kursmaterial wurde bereits während des Semesters unter dem Gesichtspunkt einer Weiterverwendung und Veröffentlichung als OER erstellt und mit weiteren Inhalten zum wissenschaftlichen Recherchieren und Schreiben unter einer CC-BY-NC Lizenz insbesondere für Incoming-Studierende kombiniert ${ }^{5}$.

Hannes Schnaitter von der HU Berlin stellte in seinem Vortrag das Konzept einer studentischen Moderation für Lehrveranstaltungen vor. Viele Lehrende weltweit haben im Sommersemester die Erfahrung gemacht, dass Studierende bei synchronen Veranstaltungen nur in den seltensten Fällen ihre Kameras aktivieren. Dies ist ein Problem, da menschliche Kommunikation zu großen Teilen auf Körpersprache, Mimik, Gestik, Intonation etc. - in der Regel aus einer Kombination von all dem - basiert. Um dies aufzufangen, wurde im weiterbildenden Masterstudiengang am IBI der HU Berlin das Konzept der studentischen Moderation entwickelt und erfolgreich umgesetzt. Eine studentische Hilfskraft übernimmt hierbei die Rolle des Moderators, ist per Video zugleich für die Lehrenden sowie die Studierenden sichtbar und interagiert in dieser spezifischen Co-Teaching-Rolle sowohl mit den Studierenden als auch den Lehrenden. In der anschließenden Diskussion wurde insbesondere thematisiert, ob dieses Co-Teaching-Konzept - vergleichbar mit dem gegenseitigen studentischen Begutachten - auch als wechselnde Rollenaufgabe von Studierenden in Seminaren wahrgenommen werden kann.

5 https://www.uni-hildesheim.de/moodle/course/view.php?id=98 [25.10.2020].
Im letzten Vortrag des Workshops berichtete Nico Saß vom IBI der HU Berlin über die studentische Evaluation des weiterbildenden Fernstudienganges „Bibliotheks- und Informationswissenschaft“. Hierbei stellt sich heraus, dass insbesondere der fehlende persönliche Kontakt zu Kommilitonen und Dozierenden, sich selbst zu motivieren, die ungewohnt (kurzen) Vorbereitungszeiten für die Livesitzungen und damit einhergehend eine persönliche Planungsunsicherheit, die grundsätzlichsten Probleme und Schwierigkeiten für dieStudierenden darstellten. Die Evaluation zeigtedarüber hinaus auch, dass eine eindeutige und klare Kommunikation der Themen, der geplanten Lernziele und der Aufgaben von zentraler Bedeutung ist und der Wechsel der verwendeten didaktischen und technologischen Elemente in Kombination mit Wiederholungen durch kleinere Tests, Quizoder auch Fragenkataloge als besonders wertvoll in der asynchronen Lehre von den Studierenden wahrgenommen wurde. An den Vortrag schloss sich eine intensive Diskussion über den Wechsel von asynchronen und synchronen Elementen an und zugleich wurde die Notwendigkeit von universitätsübergreifenden Metastudien thematisiert, um evidente Ergebnisse zu erhalten.

Die Diskussion an beiden Workshops wurde von den Teilnehmenden als lebhaft und produktiv empfunden. Zudem wurde der Komplex OER in der Informationswissenschaft als wichtiges Thema identifiziert. Insbesondere der Mangel an qualitativ hochwertigen Lehr- und Lernmaterialien, die unter freien Lizenzen nachnutzbar gestaltet und zugleich modularisiert aufgebaut sind, wurden als Desiderat benannt. HI und KIBA werden diesen Bedarf im Rahmen weiterer Workshops thematisieren.

Deskriptoren: Digitale Lehre, Studium, Informationswissenschaft, Workshop

\section{Prof. Vivien Petras, PhD}

Humboldt-Universität zu Berlin

Institut für Bibliotheks- und Informationswissenschaft

Lehr- und Forschungsbereich Information Retrieval

Dorotheenstraße 26

10117 Berlin

vivien.petras@ibi.hu-berlin.de

Prof. Dr. Stefan Schmunk

Hochschule Darmstadt,

Fachbereich Media

Max-Planck-Straße 2

64807 Dieburg

stefan.schmunk@h-da.de 